\title{
Rogue Waves of the Kundu-DNLS Equation
}

\author{
Shibao Shan, Chuanzhong Li, Jingsong He \\ Department of Mathematics, Ningbo University, Ningbo, China \\ Email: piaoxiaodan@163.com
}

Received 2013

\begin{abstract}
In this paper, we give the Lax pair and construct the Darboux transformation of the Kundu-DNLS equation. Furthermore, the rogue wave solutions of the Kundu-DNLS equation are derived by using the Taylor expansion of the breather solution. What's more, the triangular and the circular patterns of the third rouge solution are displayed.
\end{abstract}

Keywords: Kundu-DNLS Equation; Darboux Transformation; Rogue Waves

\section{Introduction}

As one of the most important integrable systems in many branches of physics and applied mathematics, the derivative nonlinear Schrl" $\{0\}$ dinger (DNLS) equation

$$
i q_{t}+q_{x x}-i \alpha\left(|q|^{2} q\right)_{x}=0
$$

has been studied in optics, water wave and so on $[1,2]$.

Considering the significance of the higher order nonlinearities in physical system, the DNLS equation yields an integrable higher nonlinear equation, i.e. Kundu-DNLS equation $[3,4]$

$$
\begin{aligned}
& i Q_{t}+Q_{x x}+i \alpha\left(Q^{2} Q^{*}\right)_{x}-\left(\theta_{t}+\theta_{x}^{2}-i \theta_{x x}\right) Q \\
& +\theta_{x}\left(2 i Q_{x}-\alpha Q^{2} Q^{*}\right)=0
\end{aligned}
$$

by means of a nonlinear transformation of the field $q \rightarrow Q=q e^{-i \theta}$ with arbitrary gauge function $\theta$.

For example, setting $\theta=\delta\left[Q\left(x^{\prime}\right)^{2} d x^{\prime}\right.$, Kundu-DNLS equation implies Eckhaus-Kundu (EK) equation.

Here $Q^{*}$ denotes the complex conjugate of $Q$, and $\alpha \mathrm{s}$ a real parameter.

Rogue waves are one of those fascinating destructive phenomena in nature that have not been fully explained so far. Understanding the initial conditions that foster rogue waves could be useful both in attempts to avoid them by seafarers and in generating highly energetic pulses in optical fibers. There are several method to solve the integrable equations, for instance, Hirota method [5], inverse scattering transformation [6], bilinear method [7], Darboux transformation [8]. In this paper, we have given the rogue waves of the DNLS and the coupled system of Hirota and Maxwell-Bloch equations $[2,9,10]$. Inspired by the importance of these recent interesting developments about the analysis of rogue waves of the NLS-type equations, we shall construct the rogue wave solutions of the Kundu-DNLS equation with the help of the Darboux transformation.

\section{Darboux Transformation and Lax Pair}

The Darboux transformation is a powerful method used to generate the soliton solutions for integrable equations. Inspired by classical Darboux transformation for the DNLS equation, we consider the coupled Kundu-DNLS equation,

$$
\begin{aligned}
& i Q_{t}+Q_{x x}-i \alpha\left(Q^{2} R\right)_{x}-\left(\theta_{t}+\theta_{x}^{2}-i \theta_{x x}\right) Q \\
& +\theta_{x}\left(2 i Q_{x}-\alpha Q^{2} R\right)=0 \\
& i R_{t}+R_{x x}-i \alpha\left(R^{2} Q\right)_{x}+\left(\theta_{t}+\theta_{x}^{2}+i \theta_{x x}\right) R \\
& +\theta_{x}\left(2 i R_{x}-\alpha R^{2} Q\right)=0
\end{aligned}
$$

where $\theta$ is a arbitrary gauge function. This form of the equation is very extensive, which is reduced to the equation (2) for $R=-Q^{*}$ with the sign of the nonlinear term changed. The Kundu-DNLS equation can be obtained if $\alpha$ is a real parameter.

We first present a general framework for deriving the required conservation rule for the DNLS equation. We start with the linear set of Lax equations:

$$
\Phi_{x}=U \Phi, \Phi_{t}=V \Phi,
$$

where $U$ and $V$ depend on the complex constant eigenvalue parameter $\lambda$.

$$
\begin{aligned}
& U=i \frac{\lambda^{2}}{4}\left(\begin{array}{cc}
1 & 0 \\
0 & -1
\end{array}\right)+\frac{i}{2} \lambda \sqrt{\alpha}\left(\begin{array}{cc}
0 & \mathrm{Re}^{-i \theta} \\
Q e^{i \theta} & 0
\end{array}\right), \\
& V=i\left(\frac{\lambda^{4}}{8}-\frac{\alpha}{4} \lambda^{2} Q R\right)\left(\begin{array}{cc}
1 & 0 \\
0 & -1
\end{array}\right)+i\left(\begin{array}{cc}
0 & G^{*} \\
G & 0
\end{array}\right),
\end{aligned}
$$

with

$G=\frac{\lambda}{4} \sqrt{\alpha}\left(-\lambda Q e^{i \theta}+2 i\left(Q_{x} e^{i \theta}+i Q e^{i \theta} \theta_{x}\right)+2 \alpha Q^{2} Q^{*} e^{i \theta}\right)$. 
where $\lambda$ is the eigenvalue, $\Phi$ is the eigenfunction corresponding to $\lambda$.

In general, considering the universality of Darboux transformation, according to the Kundu-DNLS equation (3) and (4), we can start from

$$
T=\left(\begin{array}{ll}
a_{2} & b_{2} \\
c_{2} & d_{2}
\end{array}\right) \lambda^{2}+\left(\begin{array}{ll}
a_{1} & b_{1} \\
c_{1} & d_{1}
\end{array}\right) \lambda+\left(\begin{array}{ll}
a_{0} & b_{0} \\
c_{0} & d_{0}
\end{array}\right),
$$

where $a_{0}, b_{0}, c_{0}, d_{0}, a_{1}, b_{1}, c_{1}, d_{1}, a_{2}, b_{2}, c_{2}, d_{2}$ are functions of $x, t$.

From

$$
T_{x}+T U=U^{[1]} T
$$

and

$$
T_{t}+T V=V^{[1]} T
$$

then the now solutions $\left(Q^{[1]}, R^{[1]}\right)$ are given by

$$
\begin{aligned}
& Q^{[1]}=\frac{\Omega_{21}^{2}}{\Omega_{11}^{2}} Q+\frac{\Omega_{21} \Omega_{22} e^{-i \theta}}{\Omega_{11}^{2} \sqrt{\alpha}}, \\
& R^{[1]}=\frac{\Omega_{11}^{2}}{\Omega_{21}^{2}} Q+\frac{\Omega_{11} \Omega_{12} e^{i \theta}}{\Omega_{21}^{2} \sqrt{\alpha}} .
\end{aligned}
$$

Here

$$
\begin{aligned}
& \Omega_{11}=\left|\begin{array}{ll}
\lambda_{1} \varphi_{1} & \phi_{1} \\
\lambda_{2} \varphi_{2} & \phi_{2}
\end{array}\right|, \Omega_{12}=\left|\begin{array}{ll}
\lambda_{1}^{2} \phi_{1} & \phi_{1} \\
\lambda_{2}^{2} \phi_{2} & \phi_{2}
\end{array}\right|, \\
& \Omega_{21}=\left|\begin{array}{ll}
\lambda_{1} \phi_{1} & \varphi_{1} \\
\lambda_{2} \phi_{2} & \varphi_{2}
\end{array}\right|, \Omega_{22}=\left|\begin{array}{ll}
\lambda_{1}^{2} \varphi_{1} & \varphi_{1} \\
\lambda_{2}^{2} \varphi_{2} & \varphi_{2}
\end{array}\right| .
\end{aligned}
$$

So far, we discussed about the determinant construction of one-fold Darboux transformation of KunduDNLS equation. As an application of these transformations of Kundu-DNLS equation, rogue wave solutions will be constructed in the next section.

\section{Rogue Wave Solutions}

In this section, we construct the rogue wave solution of Kundu-DNLS equation. This kind of solution only appears in some special region of time and space and then drown into a fixed non-vanishing plane. By making use of the Taylor expansion for the breather solution, one order rogue wave solution of $Q_{r}^{[1]}$ for the Kundu-DNLS equation is obtained

$$
\begin{gathered}
Q_{r}^{[1]}=\frac{-v_{1} e^{-i(2 x+t)}}{v_{2}} \\
v_{1}=3+8 x^{2}+8 i t x^{2}+8 i x t^{2}+8 x t-8 t^{2} x^{2}-4 t^{4} \\
-4 x^{4}+8 i x^{3}-4 i x+12 i t+8 i t^{3}-8 t^{2} \\
v_{2}=-1+8 i t^{2}+4 i t+8 i t x^{2}-8 i t^{2} x-8 t x \\
-8 t^{2} x^{2}-8 i x^{3}-4 t^{4}-4 x^{4}-4 i x
\end{gathered}
$$

The picture of one order rogue wave solution of the Kundu-DNLS equation and its corresponding density graph are plotted in Figure 1.

When we take $a=-2, c=1, \xi=1, \eta=1$, the picture of second rogue wave solution for the Kundu-DNLS equation is displayed in Figure 2.

Next, we examine third-order rogue waves. In this case, form the figures, We can get third-order rogue wave solution with the help of $a=-2, c=1, \alpha=1$, the picture of third-order rogue wave solution is displayed in Figure 3.

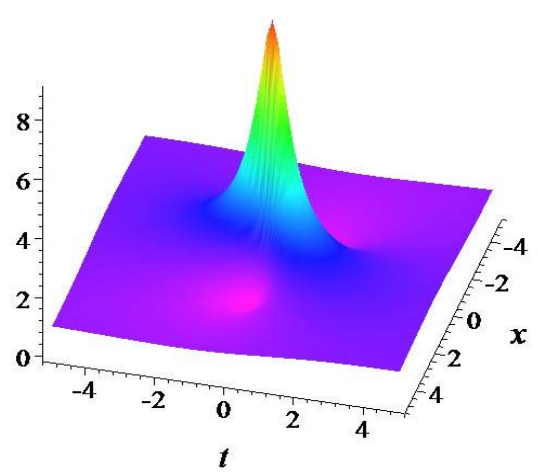

Figure 1. The One order rogue wave solution of the KunduDNLS equation with $a=-2, c=1, \xi=1, \eta=1$.

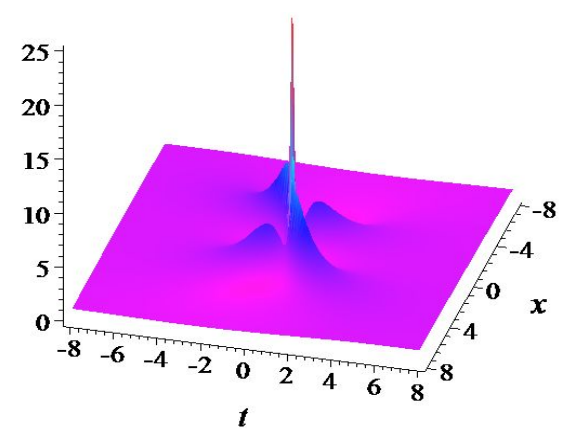

Figure 2. The second order rogue wave solution of the KunduDNLS equation with $a=-2, c=1, \xi=1, \eta=0.8$.

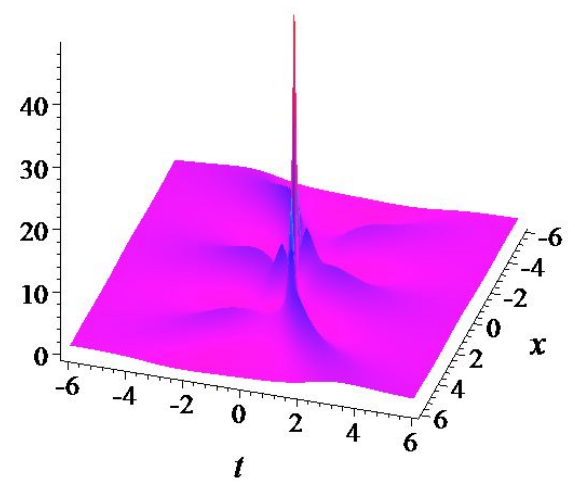

Figure 3. The third order rogue wave solution $\left|Q_{r}^{3}\right|^{2} f$ the Kundu-DNLS equation with $a=-2, c=1, \xi=0.8, \eta=0.8$. 


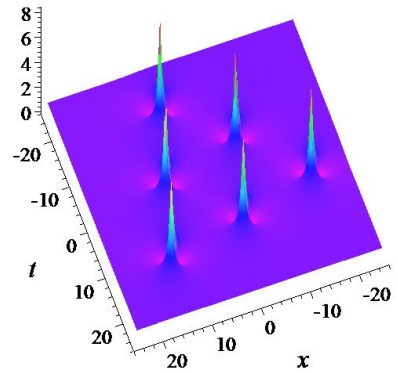

(a)

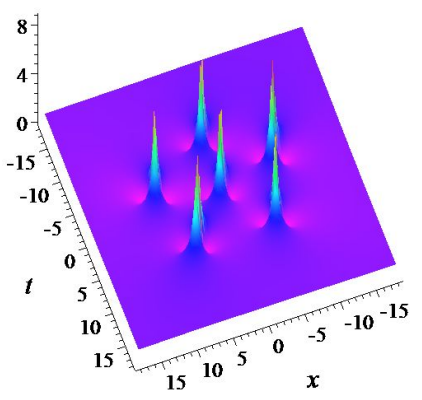

(b)

Figure 4. The third order rogue wave solution $\left|Q_{r}^{3}\right|^{2}$ of the Kundu-DNLS equation with (a) $a=-2, c=1, S_{0}=0, S_{1}=$ 500, $S_{2}=0$; (b) $a=-2, c=1, S_{0}=0, S_{1}=0, S_{2}=1000$.

We can split the third order rogue wave solution into triangle structure. A particular structure is displayed in Figure 4(a). The third-order rogue wave is seen to possess a regular triangle spatial symmetry structure.

What's more, we also can split the third order rogue wave solution into pentagon structure. A particular structure is displayed in Figure 4(b). The third-order rogue wave exhibits a regular pentagon spatial symmetry structure.

\section{Conclusions}

In this paper, we construct the Darboux transformation for the Kundu-DNLS equation. This Darboux transformation, in particular, allows us to calculate higher order rogue wave solutions in a unified way. In this way, we can derive the higher order rogue wave solutions for Kundu-DNLS equation by making use of the Darboux transformation. Particularly, these rogue wave solutions possess several free parameters. With the help of these parameters, these rogue waves constitute some patterns, such as fundamental pattern, triangular pattern, circular pattern.

\section{Acknowledgements}

This work is supported by the NSF of China under Grant No.11271210 and K. C. Wong Magna Fund in Ningbo
University. Jingsong $\mathrm{He}$ is also supported by Natural Science Foundation of Ningbo under Grant No. 2011A610179. Chuanzhong Li is supported by the National Natural Science Foundation of China under Grant No.11201251, the Natural Science Foundation of Zhejiang Province under Grant No. LY12A01007. We thank Prof. Yishen Li (USTC, Hefei, China) for his long time support and useful suggestions.

\section{REFERENCES}

[1] R. S. Johnson, "On the Modulation of Water Waves in the Neighbourhood of kh 1.363," Proceedings of the Royal Society A, Vol. 357, No. 1689,1977, pp. 131-141. doi: $10.1098 /$ rspa.1977.0159

[2] S. W. Xu, J. S. He and L. H. Wang, "The Darboux Transformation of the Derivative Nonlinear Schrodinger Equation," Journal of Physics A: Mathematical and Theoretical, Vol. 44, No. 30, 2011, p. 305203. doi: $10.1088 / 1751-8113 / 44 / 30 / 305203$

[3] A. Kundu, "Integrable Hierarchy of Higher Nonlinear Schrodinger Type Equations, Symmetry, Integrability and Geometry," Methods and Applications, Vol. 2, No. 12, 2006.

[4] A. Kundu, "Landau-Lifshitz and Higher-Order Nonlinear Systems Gauge Generated from Nonlinear schrl"\{o\}dinger Type Equations," Journal of Mathematical Physics, Vol. 25, No. 12,1984, p. 3433.

doi: $10.1063 / 1.526113$

[5] G. Tan $\backslash v\{0\}$ glu, "Hirota Method for Solving Reaction-Diffusion Equations with Generalized Nonlinearity," International Journal of Nonlinear Science, Vol. 1,2006, pp. 30-36.

[6] M. J. Ablowitz and P. A. Clarkson and Solitons, "Nonlinear Evolution Equations and Inverse Scattering," Journal of Fluid Mechanics, Vol. 244, 1992, pp.721-725.

[7] S. Kakei, N. Sasa and J. Satsuma, "Bilinearization of a Generalized Derivative Nonlinear schr $"\{0\}$ dinger Equation," Journal of the Physical Society of Japan, Vol. 64, 1995, pp.1519-1523. doi: 10.1143/JPSJ.64.1519

[8] J. S. He, L. Zhang, Y. Cheng and Y. S. Li, "Determinant representation of Darboux transformation for the AKNS System," Science in China Series A: Mathematics, Vol. 49, No. 12, 2006, pp. 1867-1878. doi: $10.1007 / \mathrm{s} 11425-006-2025-1$

[9] C. Z. Li, J. S. He and K. Porsezian, "Rogue Waves of the Hirota and the Maxwell-Bloch Equation," Physical Review E, Vol. 87, 2013, p. 012913. doi: 10.1103/PhysRevE.87.012913

[10] J. S. He, H. R. Zhang, L. H. Wang, K. Porsezian and A. S. Fokas, "A Generating Mechanism for Higher Order Rogue Waves". 